\title{
HABILIDADES AUDITIVAS E MEDIDAS DA IMITÂNCIA ACÚSTICA EM CRIANÇAS DE 4 A 6 ANOS DE IDADE
}

\author{
Auditory abilities and acoustic immittance measures \\ in children from 4 to 6 year old
}

Rafaela Della Giacoma Prado Toscano ${ }^{(1)}$, Adriana Ribeiro Tavares Anastasio (2)

\begin{abstract}
RESUMO
Objetivo: avaliar o desempenho em habilidades auditivas e as condições de orelha média de crianças de 4 a 6 anos de idade. Método: foram aplicados os testes de detecção sonora (audiômetro pediátrico em 20dBNA), a Avaliação Simplificada do Processamento Auditivo (ASPA) e as medidas de imitância acústica (handtymp com tom de $226 \mathrm{~Hz}$ ) em 61 crianças com média de idade de 5,65 anos. Para comparar os resultados das provas de habilidades auditivas e das medidas da imitância acústica foi aplicado o teste exato de Fisher com nível de significância de $p<0,05$. Resultados: houve alteração em pelo menos uma das habilidades auditivas investigadas em $24,6 \%$ das crianças. Houve alteração timpanométrica em $34,4 \%$ das crianças e $64 \%$ foram classificadas no critério "falha" para a pesquisa do reflexo acústico ispilateral. As crianças mais jovens apresentaram maior ocorrência de alterações de orelha média, mas não houve diferença estatisticamente significante entre as diferentes idades para as provas realizadas. Conclusão: as crianças mais jovens apresentaram maior ocorrência de alterações nas provas de habilidades auditivas e nas medidas de imitância acústica. Programas de investigação e acompanhamento das condições de orelha média e das habilidades auditivas em idade pré-escolar e escolar podem eliminar ou minimizar intercorrências que alterariam o desenvolvimento sócio-linguístico.
\end{abstract}

DESCRITORES: Percepção Auditiva; Memória; Localização de Som; Testes de Impedância Acústica; Triagem; Creche

\section{INTRODUÇÃO}

O desenvolvimento das diferentes habilidades auditivas depende da integridade do sistema auditivo ao nascimento e da experiência acústica no meio ambiente. Ao vivenciar sons verbais e não verbais, a criança desenvolve suas habilidades auditivas, etapas constituintes do processamento auditivo'.

$\mathrm{Na}$ fase pré-escolar há um rápido desenvolvimento nas áreas sensório-motora, cognitiva, sócio emocional e linguística ${ }^{2}$ e a ocorrência de perda auditiva periférica nesta fase pode acarretar

(1) Fonoaudióloga; Especialização em Distúrbios da Comunicação Humana pela Universidade Federal de São Paulo - UNIFESP, São Paulo, Brasil.

(2) Fonoaudióloga; Professor Doutor do Curso de Fonoaudiologia da Faculdade de Medicina de Ribeirão Preto - FMRP - USP, São Paulo, Brasil.

Conflito de interesses: inexistente inúmeras implicações, como atraso no desenvolvimento das habilidades auditivas e comunicativas.

A detecção, sensação, discriminação, localização, reconhecimento, compreensão, atenção e memória para os sons são alguns dos componentes envolvidos na percepção auditiva. O papel da percepção auditiva nos distúrbios de linguagem tem sido uma área de grandes controvérsias.

Nos últimos anos, inúmeros testes padronizados para a observação comportamental das habilidades auditivas envolvidas no processo de detecção e interpretação de eventos sonoros vêm se desenvolvendo. Alguns testes não precisam de equipamentos sofisticados e podem ser utilizados como triagem do processamento auditivo em programas de saúde do escolar, como os testes de localização sonora em cinco direções, testes de memória para sons em sequência verbal e não-verbal e pesquisa do reflexo cócleo palpebral ${ }^{3}$. 
O teste de localização sonora avalia a capacidade do indivíduo em perceber as diferenças de tempo e de intensidade do som, baseadas na integração binaural da informação acústica. A capacidade de ordenação temporal dos sons pode ser avaliada pelo teste de memória para sons em sequências verbais e não verbais ${ }^{3}$.

Estudos com crianças na fase pré escolar e escolar diagnosticadas com distúrbios da comunicação e/ou de aprendizagem evidenciaram a presença de transtorno do processamento auditivo na maioria delas, com falhas nas provas de localização sonora e memória sequencial verbal e não-verbal ${ }^{3,4}$.

As populações infantis são consideradas mais propensas ao desenvolvimento das otites médias, e nesta fase, as otites médias recorrentes podem levar a uma perda auditiva de caráter flutuante desencadeando efeitos adversos no desenvolvimento da fala, linguagem e cognitivo, sendo muitas vezes percebidos quando a criança apresenta dificuldades de aprendizagem escolar ${ }^{5,6}$.

Uma perda auditiva flutuante e/ou leve traz como consequência a perda da constância das pistas auditivas e confusão dos parâmetros acústicos em situações de fala rápida, a perda de informações de prosódia, quebra da habilidade de perceber a acústica dos sons da fala e abstração errônea das regras gramaticais Nessas condições, podem ocorrer enormes prejuízos escolares, como, dificuldades para localizar a fonte sonora, entender a fala em presença de ruído, manter a atenção e seguir instruções dadas em sala de aula ${ }^{7}$.

Durante o processo de alfabetização, deve-se priorizar a identificação precoce de perdas auditivas, os problemas de orelha média e as alterações em habilidades auditivas. Quando a relação existente entre o ouvir, o falar, o ler e o escrever, não se desenvolvem adequadamente pode levar o indivíduo a falar, ler e/ou escrever mal. Alterações em habilidades de localização sonora, memória sequencial para sons verbais e não verbais têm estreita relação com alterações de linguagem e aprendizagem ${ }^{8,9}$

O uso combinado da triagem auditiva para tons puros e medidas de imitância acústica aumenta a sensibilidade para a detecção de alterações de orelha média ${ }^{10}$. A associação de diferentes métodos de investigação das funções auditivas é a melhor maneira de detectar problemas auditivos e minimizar seus efeitos negativos na população infantil.

O objetivo deste trabalho foi avaliar o desempenho em habilidades auditivas e as condições de orelha média de crianças de 4 a 6 anos de idade.

\section{MÉTODO}

A casuística foi formada por 61 crianças com idade variando de 4 anos e 3 meses a 6 anos e 10 meses, sendo 33 crianças $(54,1 \%)$ do gênero feminino e 28 (45,9\%) do gênero masculino. Este estudo foi conduzido em uma escola privada localizada no município de Ribeirão Preto - SP. Todos os procedimentos de avaliação e orientação foram aplicados na escola, em sala silenciosa, em horários diferentes aos praticados para o intervalo de recreação das crianças para que não houvesse prejuízo das atividades didático-pedagógicas. O nível de ruído da sala foi mensurado antes do início da aplicação dos testes com o decibelímetro, modelo Minipa MSL-1350. No momento da avaliação permaneceram na sala apenas o avaliador e a criança a ser avaliada.

Como critério de inclusão, participaram desta pesquisa apenas as crianças cujos pais e/ou responsáveis assinaram o "Termo de Consentimento Livre e Esclarecido".

\section{Avaliação das habilidades auditivas}

A detecção sonora (DS) foi avaliada com o audiômetro pediátrico - modelo PA5, marca Interacoustics, com estímulo "warble" em 20dBNA apresentado a $20 \mathrm{~cm}$ do pavilhão auricular, em campo livre, nas frequências de 500, 1000, 2000 e $4000 \mathrm{~Hz}$. Iniciouse a estimulação à direita seguida da esquerda. $A$ criança foi orientada a permanecer com os olhos fechados e levantar uma das mãos ao ouvir o sinal acústico nas diferentes frequências pesquisadas. O critério "passa-falha" foi adotado para analisar cada frequência estimulada sendo "passa", quando a resposta esteve presente em 20dBNA para todas as frequências (criança levantou a mão imediatamente após o sinal acústico) e "falha" quando a resposta esteve ausente em pelo menos uma das freqüências ${ }^{10}$.

A aplicação da Avaliação Simplificada do Processamento Auditivo (ASPA) foi baseada na proposta de Pereira ${ }^{3}$, onde o teste de localização sonora (LS) foi pesquisado por meio do instrumento denominado guizo. As crianças foram orientadas a permanecerem com os olhos fechados durante a apresentação do estímulo e após o término do mesmo, deveriam apontar de onde proveio o som. O estímulo sonoro foi percutido nas seguintes posições: acima, na frente, atrás, lado direito e lado esquerdo da cabeça. Para o teste de memória sequencial não-verbal (MSNV) foram utilizados os instrumentos guizo, sino, agogô e black-black. 
Antes da avaliação propriamente dita, o avaliador apresentou os sons de cada instrumento para que a criança pudesse identificá-los. Após, foi feita uma demonstração do teste com a criança posicionada de frente para os instrumentos, e solicitado à mesma apontar a ordem de apresentação dos mesmos. Em seguida, foram apresentadas três diferentes sequências de três instrumentos para as crianças de 4 a 5 anos e, três diferentes sequências de quatro instrumentos para as crianças de 6 anos. A criança foi solicitada a apontar a sequência dos instrumentos na ordem que julgasse correta.

Para o teste de memória sequência verbal (MSV) foram utilizadas as sílabas "pa", "ta", "ca". Num primeiro momento, foi solicitada à criança a produção isolada de cada sílaba para verificar a possibilidade de produção fonoarticulatória das sílabas em questão. Após isto, o avaliador emitiu as sílabas em três diferentes sequências, sem pista visual, e a criança foi solicitada a repetir as sílabas ouvidas. As respostas foram analisadas isoladamente em relação a sua ocorrência, a partir de critérios de normalidade pré estabelecidos para a faixa etária estudada ${ }^{3}$ sendo eles, LS normal de quatro a cinco acertos; MSNV normal, acertar pelo menos duas sequências de três sons em três tentativas (4 a 5 anos) e duas sequências de quatro sons em três tentativas (6 anos) e MSV normal, apresentar de dois a três acertos.

O reflexo cócleo-palpebral (RCP) foi investigado com o instrumento denominado agogô $(109,1 \mathrm{dBA})$ e as respostas foram analisadas com relação à presença ou ausência do reflexo durante a percussão do instrumento.

\section{Avaliação das Medidas da Imitância Acústica}

As medidas da imitância acústica (timpanometria e reflexo acústico do músculo estapédio) foram realizadas com o equipamento portátil modelo MiniTymp- MT10, marca Interacoustics, com tom de prova de $226 \mathrm{~Hz}$. O volume equivalente do meato acústico externo $(\mathrm{ml})$ foi obtido à $200 \mathrm{daPa}$, a amplitude timpanométrica $(\mathrm{ml})$ foi mensurada no pico do timpanograma e o reflexo acústico ipsilateral foi pesquisado para as frequências de 500, 1000, 2000 e $4000 \mathrm{~Hz}$ a 95dBNA. O registro do reflexo acústico foi considerado presente de acordo com o manual de operação do equipamento. O critério "passa" foi adotado quando houve resposta do reflexo acústico presente em todas as frequências em 95 dBNA e "falha" quando a resposta esteve ausente em pelo menos uma das frequências.

Os valores do timpanograma foram analisados isoladamente em relação a sua ocorrência, e as respostas classificadas em adequadas ou alteradas a partir dos critérios de normalidade da
American Speech-Language-Hearing AssociationASHA (1990) ${ }^{11}$ para a faixa etária estudada, sendo o volume equivalente do meato acústico externo (Vea) de 0,4 a 1,0 ml (média de 0,7) e a amplitude timpanométrica de 0,2 a 0,9 $\mathrm{ml}$ (média de 0,5). Os timpanogramas foram classificados de acordo com Jerger ${ }^{12}$.

Este trabalho teve a aprovação do Comitê de Ética em Pesquisa da Faculdade de Medicina de Ribeirão Preto da Universidade de São Paulo, de acordo com o processo HCRP no 3862/2007.

Para comparação dos resultados das provas de habilidades auditivas e das medidas da imitância acústica foi aplicado o teste exato de Fisher. O nível de significância utilizado foi de $p<0,05$ e o valor significante foi assinalado com * .

\section{RESULTADOS}

O nível médio de ruído aferido antes do início do processo de avaliação foi de 34,02dBA (mínimo de 27,7dBA e máximo de 39,9dBA).

Os resultados foram analisados de duas formas:

- Primeira análise: divisão em três grupos de acordo com a faixa etária: Grupo GA com 15 crianças de 4 anos a 4 anos e 11 meses; Grupo GB com 23 crianças de 5 anos a 5 anos e 11 meses e, Grupo GC com 23 crianças de 6 anos a 6 anos e 11 meses.

- Segunda análise: a média de idade da população estudada foi calculada e a amostra dividida em dois grupos. O Grupo G1 foi composto por 29 crianças com idade inferior à 5,65 anos e - Grupo G2 com 32 crianças com idade superior à 5,65 anos.

\section{Primeira análise}

Todas as crianças passaram na prova de detecção sonora. Na prova de localização sonora, uma menina $(1,6 \%)$ do GA apresentou resposta alterada. Na prova de memória sequencial nãoverbal (MSNV), treze crianças $(21,3 \%)$ apresentaram respostas alteradas, das quais seis $(46,2 \%)$ foram do gênero masculino e sete $(53,8 \%)$ do gênero feminino. Das treze crianças que apresentaram respostas alteradas na prova de MSNV, cinco $(38,5 \%)$ pertenciam ao GA, duas $(15,4 \%)$ ao GB e seis $(46,2 \%)$ ao GC. Na prova de memória sequencial verbal (MSV), seis crianças apresentaram respostas alteradas, das quais, quatro $(66,6 \%)$ foram do gênero feminino e duas $(33,3 \%)$ do gênero masculino. Das seis crianças com alteração na prova de MSV, três (50\%) pertenciam ao GA, duas $(33,3 \%)$ ao GB e uma $(16,7 \%)$ ao GC. 
Todas as crianças apresentaram presença do Reflexo Cócleo Palpebral (RCP). Na Tabela 1 está apresentado o resultado do teste exato de Fischer para comparação das provas de habilidades auditivas entre os grupos formados e não foi encontrada diferença estatisticamente significante.

\section{Segunda análise}

$\mathrm{Na}$ comparação entre as crianças com idade inferior ou superior a 5,65 anos para as habilidades auditivas estudadas não houve diferença estatisticamente significante como apresentado na Tabela 2.

Tabela 1 - comparação do desempenho para as provas de habilidades auditivas entre os grupos avaliados $(n=61)$

\begin{tabular}{lccccccc}
\hline Desempenho & GA & GB & GC & Total & $\begin{array}{c}\text { Teste } \\
\text { Fisher } \\
\text { (GAXGB) }\end{array}$ & $\begin{array}{c}\text { Teste } \\
\text { Fisher } \\
\text { (GAXGC) }\end{array}$ & $\begin{array}{c}\text { Teste } \\
\text { Fisher } \\
\text { (GBXGC) }\end{array}$ \\
\hline ADEQUADO & $9(60 \%)$ & $20(87 \%)$ & $17(73,9 \%)$ & $46(75,4 \%)$ & & & \\
& & & & & $p=0.1154$ & $\mathrm{p}=0.4809$ & $\mathrm{p}=0.4591$ \\
ALTERADO & $6(40 \%)$ & $3(13 \%)$ & $6(26,1 \%)$ & $15(24,6 \%)$ & & & \\
\hline Total & $\mathbf{1 5 ( 2 4 , 6 \% )}$ & $\mathbf{2 3 ( 3 7 , 7 \% )}$ & $\mathbf{2 3 ( 3 7 , 7 \% )}$ & $\mathbf{6 1 ( 1 0 0 \% )}$ & & & \\
\hline
\end{tabular}

GA: 4 anos a 4 anos e 11 meses; GB: 5 anos a 5 anos e 11 meses; GB: 6 anos a 6 anos e 11 meses. Teste Fisher.

Tabela 2 - Comparação do desempenho para as provas de habilidades auditivas entre os grupos com idade inferior e superior à média de idade da população avaliada $(n=61)$

\begin{tabular}{lcccc}
\hline Desempenho & G1 & G2 & Total & $\begin{array}{c}\text { Teste Fisher } \\
\text { (G1XG2) }\end{array}$ \\
\hline ADEQUADO & $22(36 \%)$ & $24(39 \%)$ & $46(75,4 \%)$ & $\mathrm{p}=1.0000$ \\
ALTERADO & $7(11 \%)$ & $8(13 \%)$ & $15(24,6 \%)$ & \\
\hline Total & $\mathbf{2 9}(\mathbf{4 8 \% )}$ & $\mathbf{3 2}(\mathbf{5 2} \%)$ & $\mathbf{6 1}(\mathbf{1 0 0 \% )}$ & \\
\hline
\end{tabular}

G1: crianças com idade abaixo da média de idade da amostra; G2: crianças com idade acima da média de idade da amostra. Teste Fisher.

\section{Imitância Acústica}

Os dados da imitância acústica foram analisados de acordo com a divisão de grupos da primeira análise. Nas crianças do GA, a curva tipo "B" bilateral esteve presente em uma $(6,7 \%)$ criança e unilateral em três (20\%); a curva tipo "C" bilateral foi encontrada em uma $(6,7 \%)$ criança e unilateral em três $(20 \%)$. Duas $(13,3 \%)$ crianças apresentaram volume equivalente do meato acústico externo (Vea) acima de $1,0 \mathrm{ml}$ e em quatro (26,7\%), a amplitude timpanométrica foi inferior a 0,2 ml. Onze (73,3\%) crianças apresentaram resposta alterada no reflexo acústico ipsilateral a 95dBNA, sendo duas (13,3\%) com alteração unilateral e nove $(60 \%)$ bilateral.

No GB, duas $(8,7 \%)$ crianças apresentaram curva tipo "B" bilateral e uma $(4,3 \%)$ unilateral; a curva tipo "As" foi unilateral para uma $(4,3 \%)$ criança. Em cinco $(21,7 \%)$ crianças houve a presença da curva tipo "C", sendo bilateral em uma (4,3\%). Seis $(26,1 \%)$ crianças apresentaram volume equivalente do meato acústico externo (Vea) acima de 1,0 ml e em quatro (17.4\%), a amplitude timpanométrica foi inferior a 0,2 ml. Treze $(56,5 \%)$ crianças apresentaram alteração no reflexo acústico, sendo nove $(39,1 \%)$ com alteração bilateral.

Para o GC, três $(13,0 \%)$ crianças apresentaram curva tipo "B", e três $(13,0 \%)$ curva tipo "C". Quatro $(17,4 \%)$ apresentaram amplitude timpanométrica inferior a $0,2 \mathrm{ml}$ e duas $(8,7 \%)$ apresentaram Vea maior que $1,0 \mathrm{ml}$. Quinze $(65,2 \%)$ crianças apresentaram alteração no reflexo acústico, sendo cinco $(21,7 \%)$ com alteração bilateral. Não houve diferença estatisticamente significante na comparação das medidas de imitância acústica entre os grupos (Tabelas 3 e 4). 
Tabela 3 - Comparação do desempenho para a timpanometria entre os grupos avaliados $(n=61)$

\begin{tabular}{lccccccc}
\hline Desempenho & GA & GB & GC & Total & $\begin{array}{c}\text { Teste } \\
\text { Fisher } \\
\text { (GAXGB) }\end{array}$ & $\begin{array}{c}\text { Teste } \\
\text { Fisher } \\
\text { (GAXGC) }\end{array}$ & $\begin{array}{c}\text { Teste } \\
\text { Fisher } \\
\text { (GBXGC) }\end{array}$ \\
\hline ADEQUADO & $9(60 \%)$ & $14(60,9 \%)$ & $17(73,9 \%)$ & $40(65,6 \%)$ & & & \\
ALTERADO & $6(40 \%)$ & $9(39,1 \%)$ & $6(26,1 \%)$ & $21(34,4 \%)$ & & & \\
\hline Total & $\mathbf{1 5 ( 2 4 , 6 \% )}$ & $\mathbf{2 3 ( 3 7 , 7 \% )}$ & $\mathbf{2 3 ( 3 7 , 7 \% )}$ & $\mathbf{6 1 ( 1 0 0 \% )}$ & & & \\
\hline
\end{tabular}

GA: 4 anos a 4 anos e 11 meses; GB: 5 anos a 5 anos e 11 meses; GB: 6 anos a 6 anos e 11 meses. Teste Fisher.

Tabela 4 - Comparação do desempenho para a pesquisa do reflexo acústico entre os grupos avaliados $(n=61)$

\begin{tabular}{lccccccc}
\hline Desempenho & GA & GB & GC & Total & $\begin{array}{c}\text { Teste } \\
\text { Fisher } \\
\text { (GAXGB) }\end{array}$ & $\begin{array}{c}\text { Teste } \\
\text { Fisher } \\
\text { (GAXGC) }\end{array}$ & $\begin{array}{c}\text { Teste } \\
\text { Fisher } \\
\text { (GBXGC) }\end{array}$ \\
\hline ADEQUADO & $4(26,7 \%)$ & $10(43,5 \%)$ & $8(34,8 \%)$ & $22(36 \%)$ & & & \\
ALTERADO & $11(73,3 \%)$ & $13(56,5 \%)$ & $15(65,2 \%)$ & $39(64 \%)$ & & & \\
\hline Total & $\mathbf{1 5 ( 2 4 , 6 \% )}$ & $\mathbf{2 3}(\mathbf{3 7 , 7} \%)$ & $\mathbf{2 3}(\mathbf{3 7 , 7 \% )}$ & $\mathbf{6 1 ( 1 0 0 \% )}$ & & & \\
\hline
\end{tabular}

GA: 4 anos a 4 anos e 11 meses; GB: 5 anos a 5 anos e 11 meses; GB: 6 anos a 6 anos e 11 meses. Teste Fisher.

A Tabela 5 mostra a comparação, pelo teste exato de Fisher, da análise conjunta das provas de habilidades auditivas e imitância acústica entre os grupos avaliados ( $n=61$ ) e não foram encontradas diferenças estatisticamente significantes.

Nove crianças $(14,8 \%)$ com alteração nas provas de habilidades auditivas apresentaram alteração no reflexo acústico ipsilateral na ausência de alterações timpanométricas. Vinte $(32,8 \%)$ crianças com respostas adequadas nas provas de habilidades auditivas apresentaram alteração no reflexo acústico ipsilateral na ausência de alterações timpanométricas.

Tabela 5 - Comparação da análise conjunta do desempenho das provas de habilidade auditiva e imitância acústica entre os grupos avaliados $(n=20)$

\begin{tabular}{lccccccc}
\hline Desempenho & GA & GB & GC & Total & $\begin{array}{c}\text { Teste } \\
\text { Fisher } \\
\text { (GAXGB) }\end{array}$ & $\begin{array}{c}\text { Teste } \\
\text { Fisher } \\
\text { (GAXGC) }\end{array}$ & $\begin{array}{c}\text { Teste } \\
\text { Fisher } \\
\text { (GBXGC) }\end{array}$ \\
\hline ADEQUADO & $3(75 \%)$ & $7(87,5 \%)$ & $7(87,5 \%)$ & $17(85 \%)$ & & & \\
ALTERADO & $1(25 \%)$ & $1(12,5 \%)$ & $1(12,5 \%)$ & $3(15 \%)$ & & & \\
\hline Total & $\mathbf{4 ( 2 0 \% )}$ & $\mathbf{8 ( 4 0 \% )}$ & $\mathbf{8 ( 4 0 \% )}$ & $\mathbf{2 0 ( 1 0 0 \% )}$ & & & \\
\hline
\end{tabular}

GA: 4 anos a 4 anos e 11 meses; GB: 5 anos a 5 anos e 11 meses; GB: 6 anos a 6 anos e 11 meses. Teste Fisher.

\section{DISCUSSÃO}

A triagem auditiva em idade pré-escolar e escolar é justificada pela prevalência de perda auditiva nessa população, pelas conseqüências educacionais resultantes dessa perda e pelo prognóstico de reabilitação quando a intervenção é precoce ${ }^{7,10}$. A American Speech-Language-Hearing Association (ASHA) recomendou a triagem auditiva anual para escolares a partir dos três anos de idade 
desde $1985^{13}$, utilizando como critérios a pesquisa dos limiares tonais para as frequências de 1000, 2000 e $4000 \mathrm{~Hz}$, a 20dBNA e inclusão da frequência de $500 \mathrm{~Hz}$, se as medidas da imitância acústica não forem realizadas e o nível de ruído ambiental o permitir. O ambiente escolar não é considerado o mais propício para investigação da sensibilidade auditiva, pela presença de ruído excessivo no local. Para as triagens auditivas escolares envolvendo a pesquisa da detecção sonora, a escolha do local da triagem e o nível de ruído devem ser considerados fatores determinantes para o sucesso do teste de detecção. Neste estudo, o ruído médio na sala de teste foi de 34,02 dBA, considerado adequado, pois a recomendação é que o valor do ruído na salateste não ultrapasse 41,5 dBNPS ${ }^{10}$ quando incluída a frequência de $500 \mathrm{~Hz}$. O decibelímetro deve ser utilizado para aferir a intensidade do ruído ambiente antes de cada prova aplicada, pois melhora a credibilidade da investigação da sensibilidade auditiva no ambiente escolar.

Todas as crianças passaram pela prova de detecção sonora e apenas uma falhou na prova de localização sonora. No final do primeiro ano de vida, a criança já é capaz de responder para tons puros em intensidade de 20 dBNA e, quando solicitada, pode apontar o local de origem do estímulo sonoro à direita, à esquerda e em uma das três direções (acima, à frente e atrás) a partir dos três anos de idade'.

A metodologia utilizada nesse trabalho (uso do audiômetro pediátrico em campo livre) não permitiu a análise das orelhas em separado, porém a detecção sonora para 20dBNA permite inferir que a sensibilidade auditiva para a melhor orelha nas frequências denominadas "freqüências da fala" encontra-se dentro da normalidade. A alteração na prova de localização sonora ocorreu em uma criança do grupo mais jovem. Nesse caso, o re-teste deve ser realizado no mesmo dia ou em curto espaço de tempo. Em caso de persistência dessa falha, a criança deverá ser encaminhada para uma avaliação audiológica ${ }^{13 .}$

Em estudo com metodologia semelhante, porém com crianças de cinco a 10 anos de idade, os autores observaram que a média de acertos para o teste de localização sonora em cinco direções encontrava-se dentro dos padrões de normalidade, entretanto, com relação à faixa etária, houve diferença estatisticamente significante no desempenho das crianças, sendo os melhores resultados obtidos com as crianças mais velhas ${ }^{14}$.

As habilidades auditivas de MSNV e MSV estiveram, respectivamente, alteradas em $21,3 \%$ e $9,8 \%$ das crianças avaliadas. No teste de MSNV, o grupo de maior faixa etária, onde o teste apresenta maior complexidade, apresentou maior ocorrência de falhas. No teste de MSV, de forma distinta, a maior ocorrência de alteração aconteceu no grupo mais jovem. A inabilidade em ordenação temporal dos sons verbais deve ser analisada com cautela. O uso de sílabas sem sentido fornece pouca informação linguística, e crianças mais jovens ainda não têm o domínio das regras da língua. Com o aumento da idade, houve melhor desempenho nesta prova.

Em estudo comparando o desempenho de crianças de 3 a 12 anos, de escola pública e privada, para o teste de memória sequencia verbal, os autores observaram que há um aumento no número de acertos conforme o aumento da idade cronológica. Para três sequências de três sílabas apresentadas, apenas as crianças com mais de nove anos foram capazes de repetir corretamente todas as sequências ${ }^{15}$.

O teste de memória para sons em sequência realizado com sons não linguísticos ou com sons verbais silábicos pode ser aplicado em crianças a partir dos três anos de idade, entretanto, a partir de seis anos de idade, há uma maior complexidade para o teste de MSNV, com o aumento do número de instrumentos sonoros utilizados ${ }^{3}$ o que poderia justificar a alteração encontrada na prova de MSNV para as crianças mais velhas.

Quinze crianças $(24,6 \%)$ apresentaram alteração em pelo menos uma prova de habilidade auditiva, sendo as alterações nas provas de MSV e MSNV as de maior ocorrência $(31,1 \%)$. Analisando isoladamente os grupos divididos por faixa etária (GA, GB e GC), houve maior ocorrência de alterações nas habilidades auditivas para as crianças mais jovens (16,4\%). Para verificar possíveis diferenças entre as crianças abaixo e acima da média de idade total, a casuística estudada foi novamente dividida em outros dois grupos, G1 e G2, respectivamente. Não houve diferença estatisticamente significante entre os grupos para as provas de habilidades auditivas (Tabelas 1 e 2).

Em estudos semelhantes, as crianças mais jovens apresentaram um percentual de falha maior quando comparadas com as mais velhas, o que enfatiza a importância do acompanhamento das habilidades auditivas durante o período pré- escolar e escolar. As habilidades auditivas desenvolvem-se com o aumento da idade, e o desempenho para os testes de memória para sons em sequência, tanto verbais quanto não verbais melhoram em função da idade cronológica ${ }^{14,15}$.

A Avaliação Simplificada do Processamento Auditivo (ASPA) é um procedimento simples e alguns autores têm recomendado sua inclusão em programas de saúde do escolar, à medida que estudos demonstraram que crianças com idade 
entre 4 e 8 anos com distúrbios de comunicação e de aprendizagem apresentaram alterações em provas de localização sonora, memória sequencial verbal e não-verbal ${ }^{3,4,16}$.

O objetivo desse estudo não foi correlacionar alterações nas habilidades auditivas com possíveis alterações de fala e/ou linguagem, mas corroborar o fato de que a atuação do fonoaudiólogo no âmbito escolar, identificando precocemente indicadores de alteração no processamento auditivo, é possível. Esta atuação é viável e necessária, pois crianças com maior defasagem nas etapas da maturação auditiva podem apresentar também maior frequência de desvios de linguagem ${ }^{17}$.

A avaliação simplificada do processamento auditivo foi utilizada como um dos instrumentos para verificar as relações entre processamento auditivo e desvio fonológico. Todas as crianças com diagnóstico de desvio fonológico apresentaram pelo menos um subperfil (decodificação, integração, função não-verbal, associação e organização de saída) do processamento auditivo alterado. Os autores concluíram que há relação entre processamento auditivo e desvio fonológico, salientado que crianças que realizaram processos fonológicos interferindo na estrutura da sílaba e processos de substituição apresentaram mais alterações no processamento auditivo ${ }^{18}$.

Os transtornos do processamento auditivo vêm sendo apontados como um dos fatores da dificuldade de aprendizagem ${ }^{19}$ e alguns autores compararam o desempenho na ASPA em crianças com e sem alterações de aprendizagem da leitura e escrita. As crianças com dificuldades de aprendizagem apresentaram maior número de alterações na ASPA, com maior número de alterações no teste de memória sequencial verbal, seguido do teste de memória sequencial não verbal e teste de localização sonora ${ }^{20,21}$.

No presente estudo, as alterações de maior ocorrência foram nas provas de MSV e MSNV, o que corrobora com a importância da triagem auditiva periférica e central nas escolas como fator importante de detecção precoce de alterações de linguagem e aprendizagem.

Diversos autores ${ }^{3,4,16}$ utilizam a pesquisa do Reflexo Cócleo-Palpebral (RCP) para investigar possíveis alterações do processamento auditivo, pois a ausência desse reflexo seria indicativa dessa alteração. Tal ausência não deve ser analisada de forma isolada ou como único indicador de alteração no processamento auditivo. Neste estudo, todas as crianças avaliadas apresentaram presença do RCP, entretanto, $24,6 \%$ apresentaram alterações em pelo menos uma das habilidades auditivas estudadas.
Aproximadamente $80 \%$ das crianças em idade pré-escolar ou escolar podem sofrer de alguma perda auditiva temporária durante 0 ano letivo, ocasionando alterações na qualidade de audição $0^{5-7,22}$.

$\mathrm{Na}$ população estudada, 21 crianças $(34,4 \%)$ apresentaram alteração timpanométrica e 39 (64\%) foram classificadas no critério "falha" para a pesquisa do reflexo acústico ispilateral. Analisando isoladamente os grupos por faixa etária, foi observado que nas crianças mais jovens essa ocorrência foi maior, mas não houve diferença estatisticamente significante (Tabelas 3 e 4).

Não houve associação entre as alterações nas provas de habilidades auditivas e alterações nas medidas de imitância acústica (Tabela 5).

Mesmo com algumas controvérsias, há estudos que relacionam alterações em algumas habilidades do processamento auditivo com alterações na ocorrência e padrão dos reflexos acústicos ${ }^{23,24}$.

Alguns autores consideram que o reflexo acústico em orelhas normais deve ocorrer para intensidades até $90 \mathrm{dBNA}^{25}$. A ausência do reflexo acústico sem sinais aparentes de comprometimento tímpano ossicular em indivíduos com alteração em habilidades auditivas deve ser melhor investigada. Neste estudo, $14,8 \%$ das crianças de 4 a 6 anos de idade apresentaram alteração em provas de habilidades auditivas, associado à alteração do reflexo acústico porém, com timpanometria adequada.

Nos programas de triagem escolar, o uso combinado das medidas de imitância acústica com provas de habilidades auditivas aumenta a sensibilidade na identificação de crianças com pequenas alterações, favorecendo a realização de encaminhamentos e orientações necessárias ${ }^{26}$.

Durante a pré-alfabetização deve-se atentar a um desempenho inferior para habilidades auditivas esperadas para cada faixa etária, pois alterações nessas habilidades podem indicar baixo desempenho escolar em longo prazo.

Na população estudada, $64 \%$ das crianças apresentaram alteração na pesquisa do reflexo acústico ipsilateral, 34,4\% apresentaram alteração timpanométrica e $24,6 \%$ apresentaram alteração em pelo menos uma das habilidades auditivas investigadas.

O desempenho inferior ao esperado deve alertar a possíveis dificuldades perceptuais que possam ocorrer em crianças aparentemente sem queixas específicas e merece melhor investigação a fim de se descartar ou evidenciar alterações que necessitem de intervenção.

As crianças que apresentaram alterações em pelo menos um dos procedimentos aplicados foram reavaliadas em prazo não superior a três meses e, no caso de persistência da alteração, os responsáveis 
receberam orientação para procurar atendimento especializado (médico e/ou fonoaudiológico).

\section{CONCLUSÃO}

$\mathrm{Na}$ população investigada, as crianças mais jovens apresentaram maior ocorrência de alterações nas provas de habilidades auditivas e nas medidas de imitância acústica. A implantação de programas de investigação e acompanhamento das habilidades auditivas e das condições de orelha média em idade pré-escolar e escolar pode cursar com condutas adequadas para eliminar ou minimizar intercorrências que alterariam o desenvolvimento sócio-linguístico.

\section{AGRADECIMENTOS}

Agradecemos aos pais por consentirem o estudo e à direção da escola pela disponibilização de local adequado à triagem.

Agradecimento à Fundação de Amparo à Pesquisa do Estado de São Paulo pelo auxílio recebido para execução do estudo.

\begin{abstract}
Purpose: to evaluate the performance of auditory abilities and conditions in the middle ear of children from 4 to 6 year old. Method: we applied the tests in order to detect sound (pediatric audiometer in $20 \mathrm{dBHL}$ ), the Simplified Assessment of Auditory Processing (SAAP) and the acoustic immittance measures (handtymp with a probe of $226 \mathrm{~Hz}$ ). In order to compare the results from the tests of auditory abilities and measures of acoustic impedance we applied Fisher's exact test with significance level of $p<0.05$. Results: the study involved 61 children with a mean age of 5.65 years. There was some alteration in at least one of the investigated auditory skills in $24.6 \%$ of the children. Tympanometry was altered in $34.4 \%$ of the children and $64 \%$ were classified on the "fail" criterion for the measure of ipsilateral acoustic reflex. Younger children had higher incidence of middle ear disorders, but with no significant statistical difference between different ages as for the tests. Conclusion: younger children showed a higher occurrence of alterations in the tests of auditory abilities and measures of acoustic immittance. Research and monitoring programs for the conditions of the middle ear and auditory skills in preschool and school age can eliminate or minimize events that would alter the socio-linguistic development.
\end{abstract}

KEYWORDS: Auditory Perception; Memory; Sound Localization; Acoustic Impedance Tests; Triage; Child Day Care Centers

\section{REFERÊNCIAS}

1. Pereira LD, Cavadas M. Processamento auditivo central. In: Frota S. Fundamentos em Fonoaudiologia: Audiologia. Rio de Janeiro: Guanabara Koogan; 1998. p.135-46.

2. Boothroyd A. Management of hearing loss in children: no simple solutions. In: Seewald RC. A sound foundation through early amplification. USA: Phonak; 2000. p. 1- 12.

3. Pereira LD. Processamento Auditivo. Temas sobre Desenvolvimento. 1993; 2 (11): 7-14.

4. Ribas A. Uma análise sobre a percepção auditiva em crianças com e sem atraso no desenvolvimento da linguagem. J. Bras. Fonoaudiol, 2000; 1 (5): 60-5.

5. American Academy of Pediatrics, Subcommittee on Otitis Media with effusion, American Academy of Otolaryngology-Head and Neck Surgery. Otitis media with effusion. Pediatrics. 2004; 113(1): 1412-29.

6. Martins AS, Vieira, MM, Vieira MR, Pereira PCS. Estudo da relação entre respiração oral e perda auditiva. Rev. Soc. Bras. Fonoaudiol. 2006; 11 (3): 175-80.

7. Momensohn-Santos TM. Otite Média: implicações para o desenvolvimento da linguagem. In: Schochat E. Processamento Auditivo. Atualidades em fonoaudiologia. Volume II. São Paulo: Editora Lovise; 1996. p. 107-24.

8. Neves IF, Schochat E. Maturação do processamento auditivo em crianças com e sem dificuldades escolares. Pró-Fono. 2005; 17(3): 311-20.

9. Furbeta TDL, Felippe ACN. Avaliação simplificada do processamento auditivo e dificuldades de leitura e escrita. Pró-Fono. 2005; 17(1): 11-8. 
10. Barrett KA. Hearing and middle-ear screening of school-age children. In: Katz J. Handbook of clinical audiology. 4 th. Philadelphia: Lippincott Williams \& Wilkins; 1994. p. 476-89.

11. American Speech-Language-Hearing Association (ASHA) Guidelines for screening for hearing impairment and middle-ear disorders. Asha. 1990; Suppl 2 (32):17-24.

12. Jerger J. Clinical experience with impedance audiometry. Arch.Otolaryngol. Chicago. 1970; 92 (4): 311-24.

13. American Speech-Language-Hearing Association (ASHA) Committee on Audiologic Evaluation. Guidelines for identification audiometry. Asha. 1985; (27): 49-52.

14. Colella-Santos MF, Bragato GR, Martins PMF, Dias AB. Triagem auditiva em escolares de 5 a 10 anos. Rev. CEFAC. 2009; 11(4): 644-53.

15. Corona AP, Pereira LD, Ferrite S, Rossi AG. Memória sequencial verbal de três e quatro sílabas em escolares. Pró-Fono. 2005;17(1): 27-36.

16. Pereira LD, Ortiz KZ. Desordem do processamento auditivo central e distúrbios da produção fonoarticulatória. In: Lichitig I, Carvallo RMM. Audição: abordagens atuais. Carapicuíba: Pró-Fono Departamento Editorial; 1997. p. 173-86.

17. Kaminski JM, Tochetto TM, Mota HB. Maturação da função auditiva e desenvolvimento de linguagem.

Rev. Soc. Bras. Fonoaudiol. 2006; 11(1): 17-21. 18. Caumo DTM. Relação entre desvios fonológicos e processamento auditivo. Rev Soc Bras Fonoaudiol. 2009; 14(2):234-40.
19. Simon LF, Rossi AG. Triagem do processamento auditivo em escolares de 8 a 10 anos. Psicol Esc Educ. 2006;10(2):293-304.

20. Pelitero TM, Manfredi AKS, Schneck APC. Avaliação das habilidades auditivas em crianças com alterações de aprendizagem. Rev.CEFAC. ahead of print Epub June 25, 2010. Available from: http://search.scielo.org/

21. Engelman L, Ferreira MIDC. Avaliação do processamento auditivo em crianças com dificuldades de aprendizagem. Rev. Soc. Bras. Fonoaudiol. 2009; 14(1): 69-74.

22. Vieira ABC, Macedo LR de, Gonçalves DU. O diagnóstico da perda auditiva na infância. Pediatria (São Paulo). 2007; 29(1): 43-9.

23. Marotta RMB, Quintero SM, Marone SAM. Avaliação do processamento auditivo por meio do teste de reconhecimento de dissílabos em tarefa dicótica SSW em indivíduos com audição normal e ausência do reflexo acústico contralateral. Rev. Bras. Otorrinolaringol. 2002; 68 (2): 254-61.

24. Anastasio ART, Momensohn-Santos TM. Identificação de sentenças sintéticas (SSI) e reflexo acústico contralateral. Pró-Fono. 2005; 17(3): 355-66.

25. Anderson H, Wedenberg E. Audiometric identification of normal hearing carriers of genes for deafness. Acta Otolaryngologica. 1968; 65(1): 535-54.

26. Chaves AD, Gatto Cl, Tomazzetti CT, Rossi DB, Aita FS.; Malheiros MA et al. Estudo das medidas de imitância acústica em pré-escolares. Rev. Bras. Med. Otorrinolaringol. 1999; 6 (1): 20-5.
http://dx.doi.org/10.1590/S1516-18462011005000080

RECEBIDO EM: 13/11/2010

ACEITO EM: 04/03/2011

Endereço para correspondência:

Adriana Ribeiro Tavares Anastasio

Avenida Bandeirantes, 3900 - Vila Monte Alegre

Ribeirão Preto - SP

CEP: $14049-900$

E-mail: anastasi@fmrp.usp.br 